\title{
SEPARABLE STATES AND POSITIVE MAPS II
}

\author{
ERLING STØRMER
}

\begin{abstract}
Using the natural duality between linear functionals on tensor products of $C^{*}$-algebras with the trace class operators on a Hilbert space $H$ and linear maps of the $C^{*}$-algebra into $B(H)$, we give two characterizations of separability, one relating it to abelianness of the definite set of the map, and one on tensor products of nuclear and UHF $C^{*}$-algebras.
\end{abstract}

\section{Introduction}

The present paper is a continuation of [10]. We continue to study the duality of positive linear maps from a $C^{*}$-algebra $A$ into the bounded operators on a Hilbert space and linear functionals on the tensor product of $A$ with the trace class operators. The paper consists of two rather independent parts. In the first we consider the definite set $D_{\phi}$ of a map $\phi$, i.e. the Jordan subalgebra of $A$ on which $\phi$ restricts to a Jordan homomorphism. The main result states that if $\phi(A)=\phi\left(D_{\phi}\right)$, then the dual functional of $\phi$ is a separable positive functional if and only if $\phi(A)$ is an abelian $C^{*}$-algebra. This is applied to give necessary and sufficient conditions for the dual functional of the canonical projection of a von Neumann algebra onto the fixed point set of a positive unital map to be separable.

In the second part we prove a variation of the Horodecki theorem [3] characterizing separable states by positive maps in the setting of tensor products of a nuclear $C^{*}$-algebra $A$ and an UHF-algebra $B$. We show that a state $\rho$ on $A \otimes B$ is the $w^{*}$-limit of convex sums of product states if and only if the compositions $\rho \circ(\iota \otimes \psi)$ are positive for all positive maps $\psi$ of $B$ into itself, $\iota$ being the identity map on $A$.

For the reader's convenience we recall some of the concepts considered in the paper.

Let $A$ be a $C^{*}$-algebra, or just an operator system. Let $\mathscr{T}$ denote the trace class operators on a Hilbert space $H$, identified with $B(H)$ when $H$ is finite dimensional. If $B$ is another $C^{*}$-algebra let $B(A, B)\left(\right.$ resp. $\left.B(A, B)^{+}\right)$denote the bounded linear (resp. positive linear) maps of $A$ into $B$. We also use the 
notation $B(A, H)$ for $B(A, B(H))$. For the following see [10]. If $\phi \in B(A, B)$ its dual functional $\tilde{\phi}$ on the projective tensor product $A \widehat{\otimes} \mathscr{T}$ is defined by

$$
\tilde{\phi}(a \otimes b)=\operatorname{Tr}\left(\phi(a) b^{t}\right) ;
$$

Tr is the usual trace on $B(H)$, and $b^{t}$ is the transpose of $b$ with respect to a given orthonormal basis for $H$. A mapping cone is a cone $0 \neq K \subseteq B(B(H), H)^{+}$ which is closed in the topology of bounded pointwise weak convergence, and which has a dense set of maps $\alpha$ such that for all $a, b \in B(H)$ the map $x \mapsto a \alpha\left(b x b^{*}\right) a^{*}$ belongs to $K$. We say $\phi \in B(A, H)^{+}$is $K$-positive if $\tilde{\phi}$ is positive on the cone

$$
P(A, K)=\{x \in A \widehat{\otimes} \mathscr{T}: \iota \otimes \alpha(x) \geq 0 \forall \alpha \in K\} .
$$

Note that if $x \in P(A, K)$ and $\beta \in B(A, A)$ is completely positive, then

$$
(\iota \otimes \alpha)(\beta \otimes \iota(x))=(\beta \otimes \iota) \circ(\iota \otimes \alpha)(x) \geq 0,
$$

hence $\beta \otimes \imath(x) \in P(A, K)$. It follows that $x \in P(A, K)$ if and only if $x \in A \widehat{\otimes} \mathscr{T}$ and $\beta \otimes \alpha(x) \geq 0$ for all $\alpha \in K, \beta \in B(A, A)$ completely positive.

A state on the tensor product of two $C^{*}$-algebras is said to be separable if it is a norm limit of convex sums of product states. Otherwise it is called entangled. Following [4] we say a map $\phi \in B(A, H)^{+}$is entanglement breaking if it is $S(H)$-positive, where $S(H)$ is the mapping cone generated by maps of the form

$$
\psi(x)=\sum_{i=1}^{n} \omega_{i}(x) a_{i},
$$

where $\omega_{i}$ is a normal state on $B(H)$ and $a_{i} \in B(H)^{+}$. By [10], Theorem 2, see also [4], $\phi$ is entanglement breaking if and only if $\tilde{\phi}$ is a separable positive linear functional.

A linear set $J$ of self-adjoint operators is called a Jordan algebra if $a, b \in J$ implies their Jordan product $a \circ b=\frac{1}{2}(a b+b a) \in J . J$ is called a JC-algebra (resp. JW-algebra) if it is norm (resp. weakly) closed. In particular the selfadjoint part $A_{s a}$ of a $C^{*}$-algebra (resp. von Neumann algebra) is a JC-algebra (resp. JW-algebra).

If $\phi \in B(A, B)^{+}$with $A, B C^{*}$-algebras, the definite set $D_{\phi}$ of $\phi$ is the set of operators $a \in A$ such that $\phi\left(a^{*} a\right)=\phi(a)^{*} \phi(a)$. In particular the self-adjoint part $\left(D_{\phi}\right)_{s a}$ is the set of self-adjoint operators such that $\phi\left(a^{2}\right)=\phi(a)^{2}$. Then $\left(D_{\phi}\right)_{s a}$ is a JC-algebra. If $a \in D_{\phi}, b \in A$ then $\phi(a \circ b)=\phi(a) \circ \phi(b)$, see [9]. Note that in [10] we only considered the self-adjoint part of $D_{\phi}$ and called that the definite set. 
The author is indebted to M. B. Ruskai for some valuable comments.

\section{Separability and abelianness}

It was shown in [10] that a map in $B(A, H)^{+}$of the form $\phi(x)=\sum \omega_{i}(x) a_{i}$ with $\omega_{i}$ states of $A$ and $a_{i} \in B(H)^{+}$has definite set whose image by $\phi$ is an abelian $C^{*}$-algebra. In this section we shall elaborate on this and show that if $\phi \in B(A, H)^{+}$has image $\phi(A)=\phi\left(D_{\phi}\right)$ then $\tilde{\phi}$ is separable if and only if $\phi(A)$ is abelian. In the von Neumann algebra case when $\phi \in B(M, M)^{+}$is normal we apply this to the positive projection map $P_{\phi}$ onto the fixed point set $M_{\phi}$ of $\phi$ in M. But first we show some preliminary results. The first is a simplified dual characterization of decomposable and entanglement breaking maps.

Proposition 1. Let $A$ be an operator system, $H$ a Hilbert space and $\phi \in B(A, H)^{+}$. Let $t$ denote the transpose map on $B(H)$. Then we have

(i) $\phi$ is decomposable if and only if $\tilde{\phi}$ is positive on the cone

$$
\left\{x \in(A \widehat{\otimes} \mathscr{T})^{+}:(\iota \otimes t)(x) \geq 0\right\} .
$$

(ii) $\phi$ is entanglement breaking if and only if $\tilde{\phi}$ is positive on the cone

$$
\{x \in A \widehat{\otimes} \mathscr{T}:(\iota \otimes \omega)(x) \geq 0 \forall \text { normal states } \omega \text { of } B(H)\} .
$$

Proof. Ad (i). Let $K$ be the mapping cone of all decomposable maps in $B(B(H), H)$. Then $\phi$ is $K$-positive if and only if $\tilde{\phi}$ is positive on the cone

$$
\{x \in A \widehat{\otimes} \mathscr{T}:(\iota \otimes \alpha)(x) \geq 0 \forall \alpha \in K\},
$$

or, since each copositive map is of the form $\beta \circ t$ with $\beta$ completely positive, it follows that if $(\iota \otimes t)(x) \geq 0$ then $(\iota \otimes \beta \circ t)(x)=(\iota \otimes \beta) \circ(\iota \otimes t)(x) \geq 0$. Thus $\phi$ is $K$-positive if and only if $\tilde{\phi}$ is positive on the cone $\left\{x \in(A \widehat{\otimes} \mathscr{T})^{+}\right.$: $(\iota \otimes t)(x) \geq 0\}$. But then it follows from [8], Theorem 3.6, that $\phi$ is $K$ positive if and only if $\phi$ belongs to the closed cone generated by all maps $\alpha \circ \psi, \alpha \in K, \psi \in B(A, H)$ completely positive, in other words, if and only if $\phi$ is decomposable, completing the proof of (i).

Ad (ii). Assume $\tilde{\phi}$ is positive on the cone

$$
\{x \in A \widehat{\otimes} \mathscr{T}:(\iota \otimes \omega)(x) \geq 0 \forall \text { normal states } \omega \text { of } B(H)\} .
$$

If $(\iota \otimes \omega)(x) \geq 0$, then

$$
(\iota \otimes a \omega)(x)=(1 \otimes a)(\iota \otimes \omega)(x) \geq 0
$$


if $a \geq 0$, since $(1 \otimes a)$ and $(\iota \otimes \omega)(x)$ commute. Thus $(\iota \otimes \alpha)(x) \geq 0$ for all $\alpha \in S(H)$, so $\phi$ is $S(H)$-positive, hence entanglement breaking, by [10], Theorem 2. The converse is obvious. The proof is complete.

The following lemma is surely well known, but is included for completeness,

Lemma 2. Let $A$ be a unital $C^{*}$-algebra and $\phi \in B(A, H)$ unital and completely positive. Then the definite set $D_{\phi}$ is a $C^{*}$-subalgebra of $A$.

Proof. Let $\phi=V^{*} \pi V$ be the Stinespring dilation of $\phi$, where $\pi$ is a representation of $A$ on a Hilbert space $K$, and $V$ is a linear map of $H$ into $K$. Since $\phi$ is unital, $V^{*} V=1$, so $V$ is an isometry. Let $p=V V^{*}$. Then $p$ is a projection in $B(K)$ such that the map $p \pi p$ is the composition of $\phi$ with the isomorphism $x \mapsto V x V^{*}$ of $B(H)$ into $B(K)$. Thus $x \in D_{\phi}$ if and only if $x \in D_{p \pi p}$. But then $x \in\left(D_{\phi}\right)_{s a}$ if and only if

$$
p \pi(x) p \pi(x) p=(p \pi(x) p)^{2}=p \pi\left(x^{2}\right) p=p \pi(x)^{2} p,
$$

hence if and only if $p \pi(x)(1-p) \pi(x) p=0$, i.e. $p \pi(x)(1-p)=0$, hence if and only if $p \pi(x)=p \pi(x) p=(p \pi(x) p)^{*}=\pi(x) p$. Thus $x \in D_{\phi}$ if and only if $p \pi(x)=\pi(x) p$. Thus $x, y \in D_{\phi}$ implies their product $x y \in D_{\phi}$, proving the lemma.

The next lemma is closely related to Theorem 10 in [10]

Lemma 3. Let $\phi \in B(A, H)$ be unital and entanglement breaking. Then $\phi\left(D_{\phi}\right)$ is an abelian $C^{*}$-algebra.

Proof. Since an entanglement breaking map is completely positive, $D_{\phi}$ is a $C^{*}$-algebra by Lemma 2. Furthermore $\phi$ is a Jordan homomorphism on $D_{\phi}$. By [6] there are two orthogonal central projections $e$ and $f$ with sum 1 in the von Neumann algebra generated by $\phi\left(D_{\phi}\right)$ such that $\phi_{1}(a)=\phi(a) e$ is a ${ }^{*}$-homomorphism, and $\phi_{2}(a)=\phi(a) f$ is a $*$-antihomomorphism. Since $\phi$ is entanglement breaking the composition of $\phi$ with the transpose map is also entanglement breaking, hence completely positive. Thus both $\phi_{1}$ and $\phi_{2}$ are both homomorphisms and antihomomorphisms. But this is possible only if their images are abelian. The proof is complete.

By the above together with some results in [10] we can now conclude the following.

Theorem 4. Let $A$ be a unital $C^{*}$-algebra and $\phi$ a unital map in $B(A, H)^{+}$ such that $\phi(A)=\phi\left(D_{\phi}\right)$. Then the following conditions are equivalent:

(i) $\phi$ is entanglement breaking.

(ii) $\phi(A)$ is an abelian $C^{*}$-algebra.

(iii) $\tilde{\phi}$ is separable. 
Proof. By Theorem 2 in [10] (i) $\Leftrightarrow$ (iii). By Corollary 3 in [10] (ii) $\Rightarrow$ (iii), and by Lemma 3 (i) $\Rightarrow$ (ii). The proof is complete.

Let $M \subset B(H)$ be a von Neumann algebra, and let $\phi \in B(M, M)^{+}$. Then $\phi^{n} \in B(M, M)^{+}$for all $n \in \mathrm{N}$. It was shown in the proof of [1] Corollary 1.6, that if $\phi$ is normal, then then the maps $\phi_{n}=\frac{1}{n}\left(\phi+\phi^{2}+\cdots+\phi^{n}\right)$ converge in the point-ultraweak topology to a positive unital projection map $P_{\phi}$, so $P_{\phi}=\left(P_{\phi}\right)^{2}$, of $M$ onto the fixed point set $M_{\phi}=\{x \in M: \phi(x)=x\}$ of $\phi$. We call this projection map the averaging projection for $\phi$. Suppose furthermore that there exists a faithful normal state $\rho$ on $M$ such that $\rho \circ \phi=\rho$. Then $\rho \circ \phi^{n}=\rho$ for all $n$, hence $\rho \circ P_{\phi}=\rho$. It follows that $P_{\phi}$ is faithful and normal. Indeed, if $\left(a_{\alpha}\right)$ is an increasing net in $M$, and $a_{\alpha} \nearrow a$, then

$$
\rho\left(P_{\phi}(a)\right)=\rho(a)=\lim \rho\left(a_{\alpha}\right)=\lim \rho P_{\phi}\left(a_{\alpha}\right) \leq \rho P_{\phi}(a),
$$

so by faithfulness of $\rho, P_{\phi}(a)=\lim P_{\phi}\left(a_{\alpha}\right)$, so $P_{\phi}$ is normal. Similarly one shows that $P_{\phi}$ is faithful. Then by [1] Corollary 1.5, $\left(M_{\phi}\right)_{s a}=P_{\phi}\left(M_{s a}\right)$ is a JW-algebra. Furthermore $M_{\phi} \subset D_{\phi}$, for if $a \in\left(M_{\phi}\right)_{s a}$ then by the KadisonSchwarz inequality, $a^{2}=P_{\phi}\left(a^{2}\right) \geq P_{\phi}(a)^{2}=a^{2}$, so $P_{\phi}\left(a^{2}\right)=P_{\phi}(a)^{2}$. Since $P_{\phi}(M)=M_{\phi}$ we thus have $P_{\phi}(M)=P_{\phi}\left(D_{\phi}\right)$. We have therefore shown the following corollary to Theorem 4 .

Corollary 5. Let $M$ be a von Neumann algebra and $\phi$ a normal unital map in $B(M, M)^{+}$such that there exists a faithful normal state $\rho$ such that $\rho \circ \phi=\rho$. Let $P_{\phi}$ be the averaging projection for $\phi$. Then the following conditions are equivalent:

(i) $P_{\phi}$ is entanglement breaking.

(ii) $M_{\phi}=P_{\phi}(M)$ is an abelian von Neumann algebra.

(iii) $\tilde{P}_{\phi}$ is a separable positive functional.

A JC-algebra is called reversible if it is closed under symmetric products $a_{1} a_{2} \ldots a_{n}+a_{n} a_{n-1} \ldots a_{1}$ for all $n \in \mathrm{N}, a_{i} \in A$. Examples of nonreversible Jordan algebras are spin factors, which are the norm closed linear span of spin systems, i.e. 1 and self-adjoint unitaries $s_{i}, i \in I \subset \mathrm{N}$, satisfying $s_{i} s_{j}+s_{j} s_{i}=0$ for $i \neq j$, where Card $I \geq 7$. By [7], Corollary 7.3, if $P$ is a faithful positive projection of a $C^{*}$-algebra $B$ into itself, then $P$ is decomposable if and only if the image $P\left(B_{s a}\right)$ of $B_{s a}$ is a reversible JC-algebra. We get the following corollary to Corollary 5 .

Corollary 6. Let $M$ be a von Neumann algebra and $\phi \in B(M, M)^{+} a$ unital normal map. Suppose there exists a faithful normal state $\rho$ such that 
$\rho \circ \phi=\rho$. Then if the JW-algebra $\left(M_{\phi}\right)_{\text {sa }}$ is nonreversible, then $\phi$ is nondecomposable. In particular there exists $x \in(A \widehat{\otimes} \mathscr{T})^{+}$such that $(\iota \otimes t)(x) \geq 0$, while $\tilde{\phi}(x)<0$.

Proof. From the above discussion $\left(M_{\phi}\right)_{s a}$ is nonreversible if and only if $P_{\phi}$ is nondecomposable. If $\phi$ is decomposable, so is $\phi^{n}$ for all $n \in \mathrm{N}$, hence $P_{\phi}$ is decomposable, so that $\left(M_{\phi}\right)_{s a}=P_{\phi}\left(M_{s a}\right)$ is reversible, contrary to assumption. Thus $\phi$ is nondecomposable. By Proposition 1 the existence of $x$ is clear.

When $M_{\phi}$ is finite dimensional the structure of $P_{\phi}$ and its dual $\tilde{P}_{\phi}$ is described in the following more general result. Recall that the centralizer of a state $\rho$ on a $C^{*}$-algebra $A$ is the set of $a \in A$ such that $\rho(a b)=\rho(b a)$ for all $b \in A$.

Proposition 7. Let $A \subset B$ be unital $C^{*}$-algebras acting on a Hilbert space $H$ with $A$ finite dimensional abelian generated by its minimal projections $e_{1}, \ldots, e_{n}$. Let $P \in B(B, A)^{+}$be a unital projection of $B$ onto $A$, and suppose $\rho$ is a faithful state on $B$ such that $\rho \circ P=\rho$. Then we have:

(i) $e_{i}$ belongs to the centralizer of $\rho$ for all $i$.

(ii) If $\rho_{i}(a)=\rho\left(e_{i}\right)^{-1} \rho\left(e_{i} a e_{i}\right), a \in B$, then

$$
P(a)=\sum_{i=1}^{n} \rho_{i}(x) e_{i} .
$$

(iii) Let $E: B \otimes B(H) \rightarrow B \otimes B(H)$ be defined by

$$
E(x)=\sum_{i=1}^{n} \rho\left(e_{i}\right)^{-1}\left(e_{i} \otimes e_{i}^{t}\right) x\left(e_{i} \otimes e_{i}^{t}\right)
$$

Then

$$
\tilde{P}=(\rho \otimes \operatorname{Tr}) \circ E .
$$

Proof. (i) If $a \in B$ then $P\left(a e_{i}\right)=P(a) e_{i}=e_{i} P(a)=P\left(e_{i} a\right)$, hence

$$
\rho\left(e_{i} a\right)=\rho\left(P\left(e_{i} a\right)\right)=\rho\left(P\left(a e_{i}\right)\right)=\rho\left(a e_{i}\right),
$$

proving (i).

(ii) If $a \in A$ then by (i)

$$
\rho(a)=\sum_{i=1}^{n} \rho\left(e_{i} a\right)=\sum_{i=1}^{n} \rho\left(e_{i} a e_{i}\right)=\sum_{i=1}^{n} \rho\left(P\left(e_{i} a e_{i}\right)\right)=\sum_{i=1}^{n} \rho\left(e_{i} P(a) e_{i}\right) .
$$


Since $A$ is abelian generated by the $e_{i}^{\prime} s, P(a)=\sum_{i=1}^{n} \omega_{i}(a) e_{i}$ with $\omega_{i}$ a state on $B$. Thus

$$
\rho(a)=\sum_{i=1}^{n} \rho\left(e_{i}\left(\sum_{j=1}^{n} \omega_{j}(a) e_{j}\right) e_{i}\right)=\sum_{i=1}^{n} \omega_{i}(a) \rho\left(e_{i}\right) .
$$

Note that $e_{i}=P\left(e_{i}\right)=\sum_{j=1}^{n} \omega_{j}\left(e_{i}\right) e_{j}$, hence $\omega_{i}\left(e_{i}\right)=1$, so $\omega_{i}\left(e_{j}\right)=\delta_{i j}$. Thus if $a=e_{j} a e_{j}$ for $i \neq j$, then $\omega_{i}(a)=0$, and $\rho(a)=\omega_{j}(a) \rho\left(e_{j}\right)$. Let $\rho_{j}$ be as in the statement of the proposition. Then $\rho_{j}$ is a state on $B$ with support $e_{j}$, and we have

$$
P(a)=\sum_{j=1}^{n} \omega_{j}(a) e_{j}=\sum_{j=1}^{n} \omega_{j}\left(e_{j} a e_{j}\right) e_{j}=\sum_{j=1}^{n} \rho_{j}(a) e_{j},
$$

proving (ii).

(iii) If $a \otimes b \in B \widehat{\otimes} \mathscr{T}$ then

$$
\begin{aligned}
\tilde{P}(a \otimes b) & =\operatorname{Tr}\left(\sum_{i=1}^{n} \rho_{i}(a) e_{i} b^{t}\right)=\sum_{i=1}^{n} \rho_{i}(a) \operatorname{Tr}\left(e_{i} b^{t}\right)=\sum_{i=1}^{n} \rho_{i}(a) \operatorname{Tr}\left(e_{i}^{t} b\right) \\
& =\sum_{i=1}^{n} \rho\left(e_{i}\right)^{-1} \rho \otimes \operatorname{Tr}\left(\left(e_{i} \otimes e_{i}^{t}\right)(a \otimes b)\left(e_{i} \otimes e_{i}^{t}\right)\right) \\
& =\rho \otimes \operatorname{Tr} \circ E .
\end{aligned}
$$

The proof is complete.

\section{Separability in $C^{*}$-algebras}

The Horodecki Theorem states that if $M$ and $N$ are full matrix algebras, and $\rho$ is a state on $M \otimes N$ with density operator $h$, the $\rho$ is separable if and only if $\iota \otimes \psi(h) \geq 0$ for all $\psi \in B(N, M)^{+}$. We shall in the present section show a version of this when $M$ is a nuclear $C^{*}$-algebra and $N$ a UHF-algebra. In that case we cannot identify a state with a density operator, so we must reformulate the theorem. First recall that a $C^{*}$-algebra $A$ is nuclear if there exists a net of triples $\left(M_{n_{\lambda}}(\mathrm{C}), \alpha_{\lambda}, \beta_{\lambda}\right)$, where $\alpha_{\lambda}: A \rightarrow M_{n_{\lambda}}(\mathrm{C})$, and $\beta_{\lambda}: M_{n_{\lambda}}(\mathrm{C}) \rightarrow A$ are completely positive maps such that $\lim _{\lambda} \beta_{\lambda} \circ \alpha_{\lambda}(a)=a$ in norm for all $a \in A$, see [11], Ch. XV, 1. A $C^{*}$-algebra $B$ is a UHF-algebra if there is a strictly increasing sequence $\left(N_{n}\right)_{n \in \mathrm{N}}$ of $C^{*}$-subalgebras isomorphic to full matrix algebras, whose union is norm dense in $B$. With $A$ and $B$ as above $A \otimes B$ has a unique $C^{*}$-norm.

We say a state $\rho$ on the tensor product $A \otimes B$ of two $C^{*}$-algebras is weakly separable if $\rho$ is a $w^{*}$-limit of finite convex sums of product states. 
TheOREM 8. Let A be a nuclear $C^{*}$-algebra and $B$ a UHF-algebra. Let $\rho$ be a state on $A \otimes B$. Then $\rho$ is weakly separable if and only if $\rho \circ(\iota \otimes \psi) \geq 0$ for all $\psi \in B(B, B)^{+}$.

We leave it as an open problem how to extend it to more general $C^{*}$ algebras. The proof will be divided into some lemmas. One of them is the finite dimensional version of the theorem. The first lemma is essentially a restatement of Theorem 3.11 in [10]. Recall that when $H$ is finite dimensional and $A$ a $C^{*}$-algebra then $A^{+} \otimes B(H)^{+}$denotes the closed cone generated by tensors $a \otimes b$ with $a$ and $b$ positive operators in $A$ and $B(H)$ respectively.

Lemma 9. Let $K$ and $H$ be finite dimensional Hilbert spaces with $\operatorname{dim} K \leq$ $\operatorname{dim} H$. Let $A \subset B(K)$ be a $C^{*}$-algebra. Then

$$
A^{+} \otimes B(H)^{+}=\left\{x \in A \otimes B(H):(\iota \otimes \psi)(x) \geq 0, \forall \psi \in B(B(H), H)^{+}\right\}
$$

Proof. Let $\mathscr{P}$ denote the cone on the right side above. Let $\phi \in B(A, H)^{+}$ and let $E_{A}$ be the trace invariant conditional expectation of $B(K)$ onto $A$. Then $E_{A} \circ \phi$ is a positive extension of $\phi$ to a map in $B(B(K), H)^{+}$. Since $\operatorname{dim} K \leq \operatorname{dim} H, \phi$ is $B^{2}(H)^{+}$-positive by [8] Theorem 3.11, hence $\tilde{\phi}$ is positive on $\mathscr{P}$. By [8], Lemma 2.1, every positive linear functional on $A \otimes B(H)$ is of the form $\tilde{\phi}$ with $\phi \in B(A, H)^{+}$. Thus every positive linear functional on $A \otimes B(H)$ which is positive on $A^{+} \otimes B(H)^{+}$is positive on $\mathscr{P}$. Since clearly $A^{+} \otimes B(H)^{+} \subset \mathscr{P}$ the two cones are equal by the Hahn-Banach Theorem. The proof is complete.

Lemma 10. Let $A, K$ and $H$ be as in the previous lemma, and let $\rho$ be a state on $A \otimes B(H)$. Then $\rho$ is separable if and only if $\rho \circ(\iota \otimes \psi) \geq 0$ for all $\psi \in B(B(H), H)^{+}$.

Proof. If $\rho$ is separable it is clear that $\rho \circ(\iota \otimes \psi) \geq 0$ for all $\psi \in$ $B(B(H), H)^{+}$. To prove the converse let $\operatorname{Tr}$ be the trace on $A \otimes B(H)$ which is 1 on each minimal projection. If $\eta \in B(A \otimes B(H), A \otimes B(H))$ then its adjoint map $\eta^{*}$ is defined by

$$
\operatorname{Tr}(\eta(x) y)=\operatorname{Tr}\left(x \eta^{*}(y)\right) .
$$

Then $\eta$ is positive if and only if $\eta^{*}$ is positive.

Let $h$ be the density matrix for $\rho$ in $A \otimes B(H)$, and let $a \in A, b \in B(H)$. Then if $\psi \in B(B(H), H)^{+}$we have

$$
\rho \circ(\iota \otimes \psi)(a \otimes b)=\operatorname{Tr}(h(\iota \otimes \psi)(a \otimes b))=\operatorname{Tr}\left(\left(\iota \otimes \psi^{*}\right)(h)(a \otimes b)\right),
$$

hence

$$
\rho \circ(\iota \otimes \psi)(x)=\operatorname{Tr}\left(\left(\iota \otimes \psi^{*}\right)(h) x\right)
$$


for $x \in A \otimes B(H)$. Thus $\left(\iota \otimes \psi^{*}\right)(h) \geq 0$ for all $\psi^{*} \in B(B(H), H)^{+}$and hence for all $\psi \in B(B(H), H)^{+}$. By Lemma $9 h \in A^{+} \otimes B(H)^{+}$, so $\rho$ is separable, completing the proof.

The above lemma is false if $\operatorname{dim} K>\operatorname{dim} H$, even when $\operatorname{dim} K=4$ and $\operatorname{dim} H=2$ Indeed, by a result of Woronowicz and the author, see [12], each map in $B\left(M_{2}(\mathrm{C}), M_{2}(\mathrm{C})\right)^{+}$is decomposable. It follows that if $\rho \circ(\iota \otimes t) \geq 0$, then $\rho \circ(\iota \otimes \psi) \geq 0$ for all $\psi \in B\left(M_{2}(\mathrm{C}), M_{2}(\mathrm{C})\right)^{+}$, hence $\rho$ satisfies the condition of the lemma. However, there exist maps when $A=M_{4}(\mathrm{C})$ which satisfy the Peres (or the PPT) condition which are not separable, [2], hence the conclusion of the lemma is false in this case.

An easy consequence of the above lemma is the following version of the Horodecki Theorem. Note that the difference from the Horodecki Theorem is that the maps $\psi$ now map $B(H)$ into itself and not into $B(K)$. However, since $\operatorname{dim} K \leq \operatorname{dim} H$, it is easy to deduce the corollary from the Horodecki Theorem.

Corollary 11. Let $A, K$ and $H$ be as in the above lemma, and let $\rho$ be a state on $A \otimes B(H)$ with density matrix $h$. Then $\rho$ is separable if and only if $(\iota \otimes \psi)(h) \geq 0$ for all $\psi \in B(B(H), H)^{+}$.

Proof. Let $\operatorname{Tr}$ be as in the above proof. Then

$$
\begin{aligned}
(\iota \otimes \psi)(h) \geq 0 \forall \psi \in B(B(H), H)^{+} & \Leftrightarrow \operatorname{Tr}((\iota \otimes \psi)(h) x) \geq 0 \forall x \geq 0 \\
& \left.\Leftrightarrow \rho\left(\left(\iota \otimes \psi^{*}\right)(x)\right)\right) \geq 0 \forall x, \psi .
\end{aligned}
$$

By the last lemma this holds if and only if $\rho$ is separable. The proof is complete.

Proof of Theorem. If $\rho$ is a state on $A \otimes B$ which is a convex sum of product states, then clearly $\rho \circ(\iota \otimes \psi) \geq 0$ for all $\psi \in B(B, B)^{+}$. Hence if $\rho$ is a $w^{*}$-limit of convex sums of product states the same inequality holds.

Conversely assume $\rho \circ(\iota \otimes \psi) \geq 0$ for all $\psi \in B(B, B)^{+}$. We want to show $\rho$ is a $w^{*}$-limit of states which are convex sums of product states. Let $x_{1}, x_{2}, \ldots, x_{n} \in A \otimes B$ and $\varepsilon>0$. We must find a state of the form $\sum_{i=1}^{n} \lambda_{i} \rho_{i} \otimes \omega_{i}$ on $A \otimes B$ such that

$$
\left|\rho\left(x_{j}\right)-\sum_{i=1}^{n} \lambda_{i} \rho_{i} \otimes \omega_{i}\left(x_{j}\right)\right|<\varepsilon
$$

for all $j$. To accomplish this we may assume the $x_{j}$ belong to the algebraic tensor product of $A$ and $B$, hence are of the form

$$
x_{j}=\sum_{i=1}^{m_{j}} a_{j i} \otimes b_{j i}, \quad a_{j i} \in A, \quad b_{j i} \in B .
$$


Since $B$ is a UHF-algebra there exists a strictly increasing sequence $N_{i}$ of subalgebras of $B$ with $N_{i} \simeq M_{n_{i}}$ (C) such that $B$ is the norm closure of $\bigcup_{i} N_{i}$. By continuity of $\rho$ we may assume there is $k_{0}$ such that $b_{j i} \in N_{k}$ for $k \geq k_{0}$. Let $E_{k}: B \rightarrow N_{k}$ be the trace invariant conditional expectation of $B$ onto $N_{k}$. Thus each $\psi \in B\left(N_{k}, N_{k}\right)^{+}$has an extension $\psi \circ E_{k}$ in $B(B, B)^{+}$. Hence $\rho \circ(\iota \otimes \psi) \geq 0$ for all $\psi \in B\left(N_{k}, N_{k}\right)^{+}$.

Since $A$ is nuclear there exists a net of triples $\left(M_{n_{\lambda}}(\mathrm{C}), \alpha_{\lambda}, \beta_{\lambda}\right)$ such that $\alpha_{\lambda}: A \rightarrow M_{n_{\lambda}}(\mathrm{C}), \beta_{\lambda}: M_{n_{\lambda}}(\mathrm{C}) \rightarrow A$ are completely positive and

$$
\lim _{\lambda}\left\|\beta_{\lambda} \circ \alpha_{\lambda}(x)-x\right\|=0
$$

for all $x \in A$. If we can show $\rho \circ\left(\beta_{\lambda} \circ \alpha_{\lambda} \otimes E_{k}\right)$ is separable for arbitrary large $\lambda$ and $k$ such that $n_{\lambda} \leq n_{k}$, then $\rho\left(x_{j}\right)$ is arbitrarily well approximated by separable states for each $j$. But

$$
\rho \circ\left(\beta_{\lambda} \circ \alpha_{\lambda} \otimes E_{k}\right)=\rho \circ\left(\beta_{\lambda} \otimes E_{k}\right) \circ\left(\alpha_{\lambda} \otimes \iota\right) .
$$

Thus it suffices to show that $\rho \circ\left(\beta_{\lambda} \otimes E_{k}\right)$ is separable.

We have thus reduced the proof to showing that $\rho \circ\left(\beta_{\lambda} \otimes \imath\right)$ is a separable functional on $M_{n_{\lambda}}(\mathrm{C}) \otimes N_{k}$ where $n_{k} \geq n_{\lambda}$. For this let $y \in\left(M_{n_{\lambda}}(\mathrm{C}) \otimes N_{k}\right)^{+}$. Since $\beta_{\lambda}$ is completely positive $\beta_{\lambda} \otimes \iota(y) \geq 0$ in $A \otimes N_{k}$, hence by assumption, if $\psi \in B\left(N_{k}, N_{k}\right)^{+}$

$$
\rho\left(\left(\beta_{\lambda} \otimes \iota\right) \circ(\iota \otimes \psi)(y)\right)=\rho \circ(\iota \otimes \psi)\left(\beta_{\lambda} \otimes \iota(y)\right) \geq 0 .
$$

It follows that the assumptions of Lemma 10 are satisfied, hence that $\rho \circ\left(\beta_{\lambda} \otimes \iota\right)$ is separable, completing the proof of Theorem 8 .

It is obvious that a convex sum of product states on a tensor product of $C^{*}$-algebras can be extended to a similar state on a tensor product of larger algebras. This is not obvious for weakly separable states. However, we have

Corollary 12. Let $A$ be a unital nuclear $C^{*}$-algebra and $A_{0} \subset A a$ $C^{*}$-subalgebra containing the identity. Let $B$ be a UHF-algebra. The every weakly separable state on $A_{0} \otimes B$ has a weakly separable extension to $A \otimes B$.

Proof. Let $\rho$ be a weakly separable state on $A_{0} \otimes B$. Let $C$ denote the norm closed cone in $A \otimes B$ generated by all operators of the form $\iota \otimes \psi(x)$ with $x \in(A \otimes B)^{+}$and $\psi \in B(B, B)^{+}$. Then $C \cap\left(A_{0} \otimes B\right)$ is the similar cone with $x$ now in $A_{0} \otimes B$. By the first paragraph of the proof of the theorem $\rho$ is positive on $C \cap\left(A_{0} \otimes B\right)$, independently of the $C^{*}$-algebra being nuclear. To conclude the proof of the corollary, by the theorem it suffices to show that $\rho$ has an extension to $A \otimes B$ which is positive on $C$. Since $\iota \otimes \iota(x)=x$ for all $x, C \cap\left(A_{0} \otimes B\right) \supset\left(A_{0} \otimes B\right)^{+}$, which has $1 \otimes 1$ as an interior point. It follows 
by a theorem of Krein-Rutman [5], Ch. V, 5.4, that $\rho$ has an extension which is positive on $C$, completing the proof.

\section{REFERENCES}

1. Effros, E., and Størmer, E., Positive projections and Jordan structure in operator algebras, Math. Scand. 45 (1979), 127-138.

2. Horodecki, P., Separability criterion and inseparable mixed states with positive partial transposition, Phys. Lett. A 232 (1997), 333-339.

3. Horodecki, M., Horodecki, P., and Horodecki, R., Separability of mixed states: necessary and sufficient conditions, Phys. Lett. A 223 (1996), 1-8.

4. Horodecki, P., Shor, P. W., and Ruskai, M. B., Entanglement channels, quant-ph /0302031.

5. Schaefer, H. H., Topological Vector Spaces, Graduate Texts in Mathematics 3, Springer, New York 1971.

6. Størmer, E., On the Jordan structure of $C^{*}$-algebras, Trans. Amer. Math. Soc. 120 (1965), 438-447.

7. Størmer, E., Decomposition of positive projections on $C^{*}$-algebras, Math. Ann. 247 (1980), $21-41$.

8. Størmer, E., Extension of positive maps into B(H), J. Funct. Anal. 66 (1986), 235-254.

9. Størmer, E., Multiplicative properties of positive maps, Math. Scand. 100 (2007), 184-192.

10. Størmer, E., Separable states and positive maps, J. Funct. Anal. 254 (2008), 2303-2312.

11. Takesaki, M., Theory of Operator Algebras III, Encyclopaedia of Mathematical Sciences 127, Springer, Berlin 2003.

12. Woronowicz, S. L., Positive maps of low dimensional matrix algebras, Rep. Math. Phys. 10 (1976), 165-183.

DEPARTMENT OF MATHEMATICS

UNIVERSITY OF OSLO

0316 OSLO

NORWAY

E-mail: erlings@math.uio.no 